\title{
siRNA-mediated knockdown of hTDE2 retards cell cycle progression through transcriptional activation of p21
}

\author{
WEI-HUA REN ${ }^{1,2}$, CHEN-YI YANG ${ }^{1}$, XIAN-MEI YANG ${ }^{1}$ and LONG YU ${ }^{1}$ \\ ${ }^{1}$ The State Key Laboratory of Genetic Engineering, Institute of Genetics, School of Life Sciences, \\ Fudan University, Shanghai 200433; ${ }^{2}$ Central Laboratory, Luoyang Central Hospital \\ Affiliated to Zhengzhou University, He'nan, Luoyang 471009, P.R. China
}

Received November 1, 2013; Accepted November 25, 2013

DOI: $10.3892 /$ or.2014.2980

\begin{abstract}
Carcinogenesis is a very complex process involving a series of changes of tumor-related genes. Therefore, genes differentially expressed in tumors have received significant attention. Among them is the tumor differentially expressed (TDE) protein family, which shows no homologue to any other protein families and is unique to eukaryotes. The members of the TDE (also known as Serinc) family are highly conserved, showing approximately $30-80 \%$ homologue of their amino acid sequences. Previous reports have shown that both human and mice TDE/Serinc proteins are always upregulated in carcinomatous tissues. However, their precise physiological roles remain unclear. The human TDE2/Serinc1 gene was cloned by our laboratory during the screening for differentially expressed genes in hepatocarcinoma. In the present study, we knocked down the expression of TDE2 with specific siRNA fragments in two human hepatocarcinoma cell lines, and this caused cell cycle arrest at G2. Cell cycle progression is monitored and regulated by several factors. p21, the cdk inhibitor, is a key player and could be transcriptionally activated by many factors including sterol regulatory element-binding proteins (SREBPs). Previous research demonstrated that rat TDE2 could facilitate the cellular sphingolipids biosynthesis in both yeast and mammalian cells. Therefore, we further analyzed the effect of TDE2 knockdown on p21 and SREBP, and found that endogenous p21 expression was upregulated, as was that of SREBPs (-1a and 2). In conclusion, our preliminary results
\end{abstract}

Correspondence to: Dr Xian-Mei Yang or Dr Long Yu, The State Key Laboratory of Genetic Engineering, Institute of Genetics, School of Life Sciences, Fudan University, 220 Handan Road, Shanghai 200433, P.R. China

E-mail: xianmei@fudan.edu.cn

E-mail: longyu@fudan.edu.cn

Abbreviations: hTDE2, human tumor differentially expressed 2; serinc, serine incorporator; SREBP, sterol regulatory elementbinding protein; SPT, serine palmitoyltransferase

Key words: hepatocarcinoma, hTDE2, membrane lipid, serinc, p21, sterol regulatory element-binding protein indicated that TDE2 may have an effect on tumor cell growth by influencing the expression of SREBP and $\mathrm{p} 21$.

\section{Introduction}

The study of genes that are differentially expressed during tumor progression may identify key players in this complex and multistep biological process. Polyomavirus large-T antigen (PyLT-Ag) transgenic mice have been used as a model system for studying differentially expressed genes during tumorigenesis (1). With the pre- and post-adenomatous testis cell lines derived from the PyLT-Ag transgenic mice, as well as the differential display technique, a testicular tumor differentially expressed gene (TDE1) was identified to be upregulated during transformation (2-15 times compared to the control cell line), indicating a potential oncogenic property possessed by it (2-4). This gene was also independently cloned by other researchers, and was designated TMS-1, AIGPI and/or Serinc3 (5-7). Subsequently, increasingly more homologous and orthologous genes of $T D E 1$ were cloned and identified in species such as yeast, worm, fruit fly, zebrafish, mouse, rat and human, giving rise to a large TDE1/TMS protein family (also known as the Serinc family for serine incorporator) (5,7-9). This novel TDE1/TMS1/Serinc family appears in eukaryotic organisms and contains no amino acid sequence homology with other known protein families. At the same time, the TDE/Serinc proteins themselves are highly conservative, sharing between 30 and $80 \%$ homology (8). There is a total five TDE/Serinc proteins (Serinc1-5) identified in mammals while baking yeast harbors only one ortholog (TMS1). The TDE/Serinc proteins contain multiple transmembrane domains (up to 11) and often an $\mathrm{N}$-terminal signal peptide.

The characteristic multiple transmembrane domains of TDE/Serinc proteins by peptide analysis suggested that they were most closely related to proteins involved in ion-channel formation or amino acid transportation. However, all attempts to detect amino acid transport with mouse Serinc1 and Serinc3 failed (5,7). In 2005, Inuzuka et al (7) studied the Serinc function by searching the yeast proteome database for its interacting partners. Two proteins involved in serine synthesis, SER3 and YGP1, were identified to interact with TMS1 (the only Serinc protein in yeast). Yeast dihybrid experiment further demonstrated that rat Serinc1 protein could also interact with 
SER3 and YPG1, indicating the possible conservation of these interactions. Moreover, it was also shown that Serinc proteins (Serinc1, 2 and 5) could additionally enhance incorporation of serine into the membrane lipid phosphatidylserine both in prokaryotes and eukaryotes. On the other hand, sphingolipids are another class of membrane lipids that could be synthesized by all eukaryotic cells from serine and palmitoyl-CoA. The condensation of these two molecules is catalyzed by serine palmitoyltransferase (SPT), the most key limiting factor in the sphingolipid de novo biosynthetic pathway. Inuzuka et al also showed that rat Serinc1 protein facilitated the cellular sphingolipid biosynthesis in both yeast and mammalian cells, probably via interacting with and enhancing the activity of SPT. The same property was also demonstrated for Serinc 2 and 5. Results of the biochemical assays performed by Inuzuka et al indicated that the Serinc family members might play pivotal roles in the biosynthesis of membrane lipids. Indeed, besides the upregulation of Serincs observed in carcinoma tissues, it was postulated that rat Serinc5/TPO1 might be involved in myelin biogenesis based on its expression pattern (9). Furthermore, in the hippocampus of rats with seizures induced by kainite, Serinc1, 2 and 5 were found to be differentially expressed compared to that of the control rats (7). These findings suggested that Serinc proteins might participate in the plasticity of the central nervous system, perhaps affecting membrane lipid biogenesis.

The conservative nature of the TDE/Serinc proteins suggests an important biological role for this family. Indeed, their overexpression was shown to correlate with carcinogenesis. In addition to the known overexpression of mouse $T D E$ gene in testicular tumors from PyLT-Ag transgenic mice, human TDE1 (hTDE1) was also shown to be highly expressed in lung cancer tissues (10). Moreover, Player et al (11) cloned and localized human Serinc 2 to chromosome 1 in 2003. Results of both in situ hybridization and real-time PCR showed that its mRNA was also upregulated in human non-small cell lung cancer tissue. However, besides the biochemical function of these proteins postulated by Inuzuka et al, the molecular mechanism underlying their oncogenic property remains to be elucidated.

Cell growth is regulated by several factors. In addition to duplication of DNA, protein, and other cellular components, recent studies have provided insight into the involvement of lipid metabolism, including membrane lipid biogenesis (therefore affecting plasma membrane, or, more importantly, secretory vesicles), in cell-cycle progression (12-16). On the one hand, Kurat et al (15) discovered that Tg14, the yeast triacylglycerol lipase, was directly activated by $\mathrm{Cdk} 1 /$ Cdc28-dependent phosphorylation; on the other hand, sterol regulatory element-binding proteins (SREBPs), which are transcriptional factors involved in cholesterol and fatty acid synthesis, have also been shown to affect cell proliferation through accumulating cdk inhibitors such as p21 (17-19). hTDE2/Serincl located on chromosome 6, was first cloned by our group in 1998 during an investigation of genes differentially expressed in liver cancer tissues (GenBank access number AF087902; unpublished data). hTDE2/Serincl has a broad expression profile and is significantly upregulated in the hepatocarcinoma tissues examined, which is consistent with the oncogenic behaviors of other Serinc members such as Serinc2 and Serinc3. In the present study, we showed that knockdown of hTDE2/Serinc1 expression resulted in cell cycle arrest and cell growth inhibition. Moreover, downregulation of TDE2/Serinc1 upregulated SREBPs, and, eventually, expression of p21 was elevated causing cell cycle retardation.

\section{Materials and methods}

Cell lines, tissue samples and other materials. The human HCC cell lines L-02, YY-8103, SMMC-7721, QGY-7703 and Huh-7 were from Fudan University (Shanghai, China). FOCUS was from the Molecular Hepatology Laboratories, MGH Cancer Center. All other cell lines used in the present study were obtained from the American Type Culture Collection (ATCC, Manassas, VA, USA). Cells were cultured in Dulbecco's modified Eagle's medium or RPMI-1640 supplemented with $10 \%$ heat-inactivated fetal bovine serum (FBS), accordingly. The cell cultures were incubated at $37^{\circ} \mathrm{C}$ in a humidified incubator with $5 \% \mathrm{CO}_{2}$. The human multiple tissue cDNA panel was purchased from Clontech.

Paired hepatoma and non-hepatoma tissue samples were obtained from 32 hepatoma patients who had surgery at Eastern Hepatobiliary Surgery Hospital, Second Military Medical University, Shanghai, China. The hospital's Ethics Committee had approved the specimen collection procedures, and written informed consent was obtained from each patient or their relatives.

To perform hTDE2 knockdown, three gene-specific siRNA fragments (460, 895 and 1394, targeting different regions of hTDE2) as well as one non-specific fragment (N.C.) were employed to transfect the HCC cell lines. The sequences were: 5'-CUGCAGCAAUUGCAAUUAUTT-3' (sense) and 5'-AUAAUUGCAAUUGCUGCAGTT-3' (antisense) for fragment 460; 5'-GGUCAGCUAUGACCAAUGATT-3' (sense) and 5'-UCAUUGGUCAUAGCUGACCTT-3' (antisense) for fragment 895; 5'-GGCACCACUUGUUCUUACATT-3' (sense) and 5'-UGUAAGAACAAGUGGUGCCTT-3' (antisense) for fragment 1394; and 5'-UUCUCCGAACGUGU CACGUTT-3' (sense) and 5'-ACGUGACACGUUCGGAGA ATT-3' (antisense) for fragment N.C. All fragments were synthesized at Shanghai GenePharma (Shanghai, China).

Real-time quantitative PCR and semi-quantitative PCR. First, total RNA was extracted from tissues or cells using TRIzol (Invitrogen) and was reverse transcribed into cDNA (Gibco-BRL) following the manufacturer's instructions. Then, real-time PCR was performed with an iCycler iQ (Bio-Rad Laboratories) to analyze the level of target mRNAs, using the dsDNA-specific binding dye SYBR-Green Premix (Toyobo). After normalizing to GAPDH, target mRNA levels were quantified with the $\Delta \Delta C_{T}$ method. Primer sets used were: 5'-AGA TAATGAAAGGGATGGTGTC-3' (sense) and 5'-ACAGC ACGATGCCAATCCAACT-3' (antisense) for hTDE2; 5'-CG GCCGGGGGAACCCAGTT-3' (sense) and 5'-CGCAGCCG CCTCC-3' (antisense) for hSREBP1a; 5'-GAAGGCTGGAG ACCAGGAAGA-3' (sense) and 5'-CGTCCACCACCGACA GATGA-3' (antisense) for hSREBP2; 5'-TGGAGACTCTCAG GGTCGAAA-3' (sense) and 5'-AGGACTGCAGGCTTCCT GTG-3' (antisense) for p21; and 5'-AGGGCTGCTTTTA ACTCTGGT-3' (sense) and 5'-CCCCACTTGATTTTGGAG GGA-3' (antisense) for GAPDH. 
To compare the expression of hTDE2 in hepatocarcinoma and pericancerous tissue samples, semi-quantitative PCR was performed. Total RNA was extracted and reverse transcribed into cDNA as described above. According to the PCR signal generated from the internal standard $\beta 2-\mathrm{MG}$, the template amount of each cDNA pool was adjusted to give the same exponential phase signal strength after 24 cycles. PCR reactions were then performed with appropriate conditions. The final PCR samples were then subjected to electrophoresis on $1.5 \%$ agarose gels and ethidium bromide staining. After capturing digital images under UV, the densitometry value of each PCR-generated DNA fragment was measured with UVP Gelworks ID Advanced software (Version 2.51). DR (dosage ratio) was used to describe the relative expression difference between the tumor and its pericancerous tissues. DR is calculated with the following formula: DR $=\left(\right.$ Density $_{\text {tumor-hTDE } 2} /$

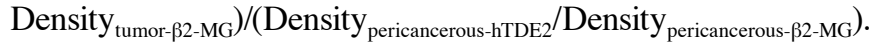
For semi-quantitative PCR, primer set 5'-TCTCTTCC AGTTGGATTGGCATC-3' (sense) and 5'-CCATAACCTAC ACTATTGTCCAC-3' (antisense) was used for hTDE2, while primer set 5'-ATGAG TATGCC TGCCGTGTGAAC-3' (sense) and 5'-TG TGGAGCAACCTGCTCAGATAC-3' (antisense) was used for $\beta 2-\mathrm{MG}$.

Cell cycle, growth curve and colony formation assay. Cells were transfected with appropriate siRNA fragments and were collected $48 \mathrm{~h}$ after transfection. Prior to flow cytometric analysis, cells were fixed with pre-chilled $\left(\right.$ at $\left.-20^{\circ} \mathrm{C}\right) 75 \%$ ethanol for $2 \mathrm{~h}$ at $4^{\circ} \mathrm{C}$, followed by washing with PBS. PI (final concentration, $50 \mu \mathrm{g} / \mathrm{ml}$ ) and RNAase (final concentration, $100 \mu \mathrm{g} / \mathrm{ml}$ ) were then added to the cell suspension and the mixture was kept for 20 min in the dark. Analysis was completed within $3 \mathrm{~h}$ after PI staining.

MTS assay was performed according to the manufacturer's instructions. The experiments were carried out in triplicate and the average absorbance was calculated to generate the growth curve.

For the colony formation assay, cells were seeded in 6-well plates as 400 cells/well, followed by culturing at $37^{\circ} \mathrm{C}$ with proper medium for 6-10 days. Every other day, old medium was replaced with fresh one. At the end of the incubation, cells were washed and fixed with $4 \%$ paraformaldehyde for $15 \mathrm{~min}$. The fixed cells were washed again and subjected to Giemsa (Sigma) staining at room temperature for $2 \mathrm{~h}$. Images of the colonies were captured after the plates were washed and dried.

Western blot analysis. Cells seeded in 6-well plates were transfected with appropriate siRNA fragments ( 50 pmole/well) using Lipofectamine $^{\text {TM }} 2000$ (Invitrogen) according to the manufacturer's instructions. Forty-eight hours after transfection, cells were lysed and processed for SDS-PAGE electrophoresis. Proteins were then transferred to nitrocellular membranes (Bio-Rad Laboratories). After blocking with 5\% milk in TBST buffer, the membranes were subjected to primary and secondary antibody incubation with washing in between and after. Membranes were then incubated in enhanced chemiluminescence (ECL) solution (Amersham) for $1 \mathrm{~min}$ followed by exposure to Hyperfilm. The primary antibodies used were: anti-human SREBP-1 polyclonal antibody (C-20, sc-366, 1:200; Santa Cruz Biotechnology), anti-human SREBP-2 polycolonal antibody (H-164, sc-5603, 1:200; Santa Cruz Biotechnology), anti human p21 monoclonal antibody (1:1,000; Abcam), anti-human p53 monoclonal antibody (1:200; Sigma) and antihuman $\beta$-actin monoclonal antibody (1:5,000; Sigma). The corresponding secondary antibodies used were all diluted as $1: 3,000$.

Luciferase constructs and luciferase assay. p21-2400-Luc and p21-221-Luc reporter plasmids were kind gifts from Dr Huang Haojie from Mayo Clinics (Rochester, MN, USA) with a pGL3-Basic backbone (Promega). In the reporter plasmids, nucleotide sequences from +73 to -221 and/or -2400 of the $p 21$ gene were cloned in front of the luciferase reporter gene. p21-2400-Luc, but not p21-221-Luc, contains two p53-binding sites. Both plasmids harbor the $S R E$ element which is located between positions -90 and -98 of the p21 promoter. The other two luciferase constructs, p21-2400-LucMut and p21-221-Luc-Mut, were generated by PCR with a site-directed mutagenesis kit (Toyobo). As a result, the wild-type $S R E$ sequence, TGGGCCGAG, was replaced by TACAAAATG (20).

To perform the luciferase assay with the dual-luciferase reporter system (Promega), cells were seeded on 24-well plates one day before transfection. Luciferase reporter plasmid $(0.1 \mu \mathrm{g})$ and pRL-SV40, the Renilla luciferase internal control plasmid $(0.01 \mu \mathrm{g}$; Promega), were co-transfected together with appropriate siRNA fragments into the cells using Lipofectamine 2000 kit (Invitrogen). Luciferase activity in each transfected sample was examined and normalized to that of the Renilla luciferase activity.

Statistical analysis. The values are expressed as means \pm SD. The Student's t-test was used to assess the differences. $\mathrm{P}<0.05$ was considered to indicate a statistically significant result.

\section{Results}

hTDE2 gene is upregulated in hepatocarcinoma tissues. hTDE2 (hSerinc1) belongs to the Serinc protein family which was highly conserved from fungi to vertebrates. Previous research indicated that high expression of these family members was correlated with carcinogenesis $(4,10,11)$. However, the physiological function of this family remains unclear. In the present study, we first assessed the expression profile of $h T D E 2$ in different human tissues with a human multiple tissue cDNA panel. The quantitative PCR results indicated that the tissue expression profile of $h T D E 2$ is broad, with the highest expression in the brain and the lowest in the thymus (Fig. 1A).

To further analyze whether hTDE2 was also correlated with hepatocarcinogenesis, we measured hTDE2 expression in 32 paired HCC and corresponding non-HCC neighboring tissue samples with semi-quantitative PCR. In 6/32 paired samples, hTDE2 was downregulated in the HCC tumor tissues; in 23 paired samples $(23 / 32=71.9 \%)$, clear upregulation of hTDE2 was observed in the tumor tissues. The remaining 3 paired samples showed a similar expression of hTDE2 in the tumor and normal tissues (Fig. 1B). This result is consistent with the previous findings that a correlation exists between upregulation of TDE proteins and carcinogenesis. 
A

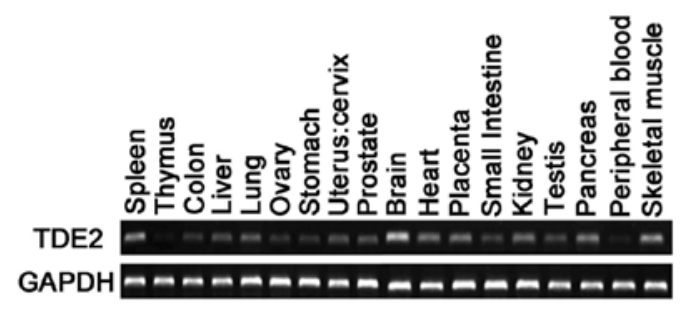

B

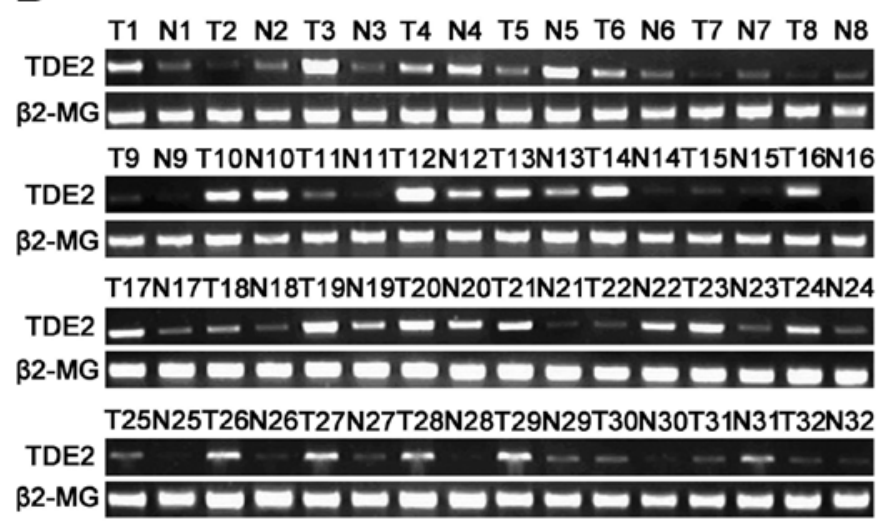

C

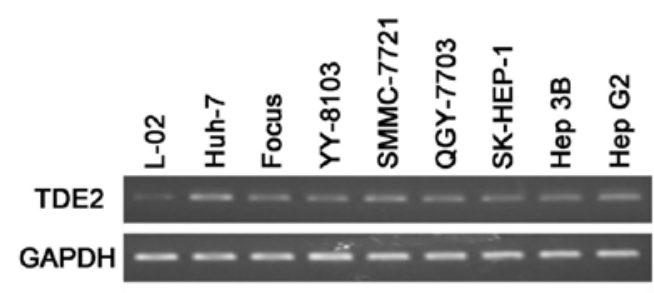

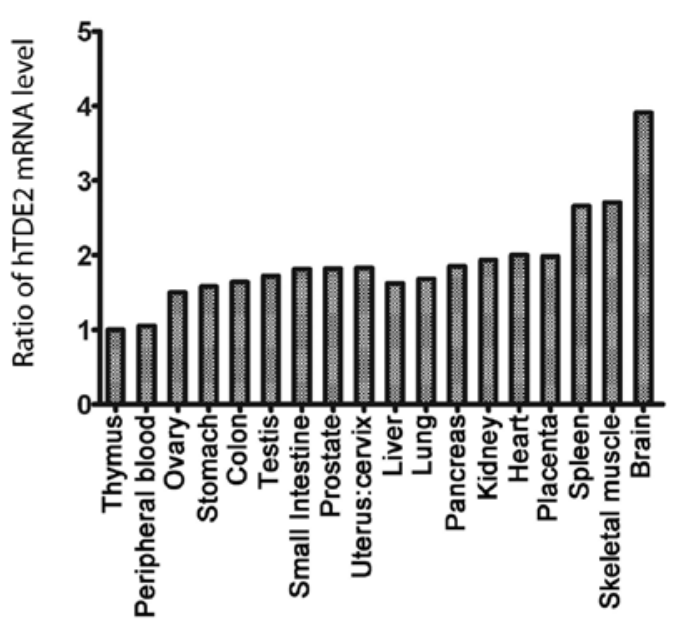
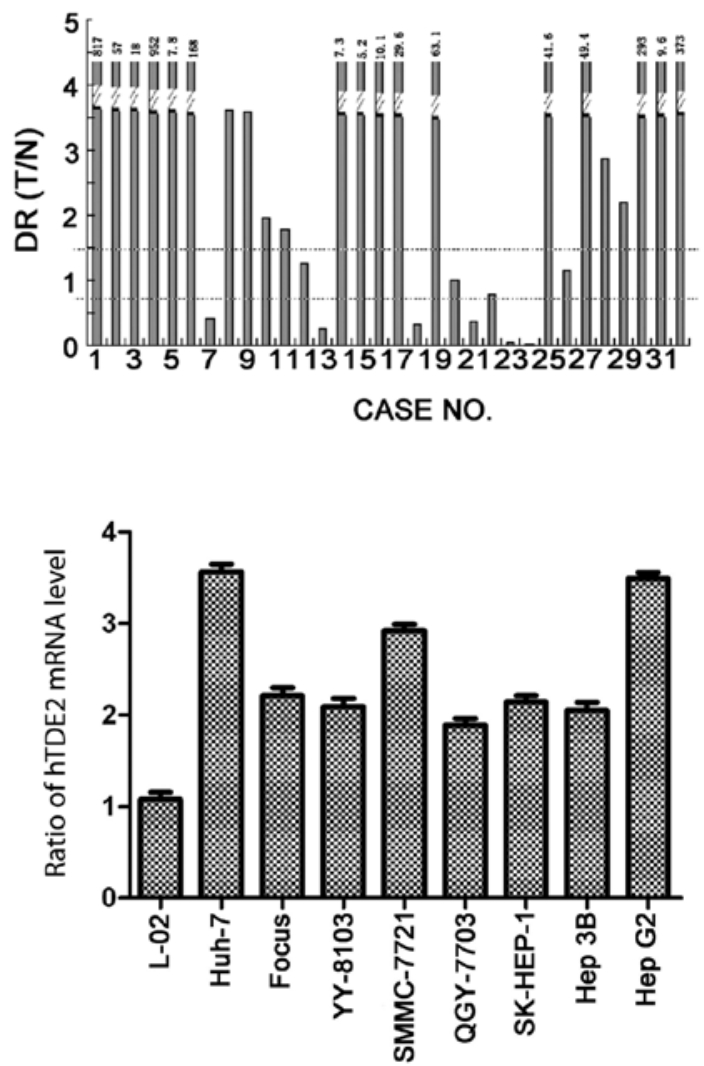

Figure 1. Expression analysis of hTDE2 in human tissues, HCC samples and cell lines. (A) Quantitative detection of $h T D E 2$ mRNA levels in 18 human tissues by real-time PCR (SYBR) using GAPDH as internal control. Ratio of $h T D E 2$ mRNA expression in various tissues to thymus is shown on the right. One tenth of the final PCR products was subjected to agarose electrophoresis (left). (B) The mRNA level of $h T D E 2$ in 32 paired human HCC (T1-32) and corresponding non-cancerous tissues (N1-32) by the semi-quantitative PCR method. A dosage ratio (DR) of $\leq 0.75$ was considered as downregulation of $h T D E 2$ in the HCC samples, while DR of $\geq 1.50$ was considered upregulation. (C) $h T D E 2$ mRNA levels in human HCC cell lines, as well as L02, by real-time PCR (SYBR). Right panel shows the ratio of hTDE2 mRNA levels in various cell lines to L02 cells.

We also measured the $h T D E 2$ mRNA level in different live cell lines commonly used in our laboratory, and found that all the cell lines expressed this gene and its expression was lower in L-02 compared to other cell lines (hepatocarcinoma lines) examined here (Fig. 1C). Since L-02 was generally considered to be a normal liver cell line, this result was consistent with previous ones with human tissue cDNA panel and HCC samples. We selected two of the hepatocarcinoma cell lines, QGY-7703 and SMMC-7721, to carry out the following study.
Knockdown of hTDE2 hinders the growth of hepatocarcinoma cells and arrests the cell cycle at $G 2$. Since $h T D E 2$ was significantly upregulated in the HCC specimens, we investigated whether knocking down its expression could have any effect on tumor cell growth. Therefore, we designed three siRNA fragments specifically targeting $h T D E 2$ expression (siRNA 460 , 895 and 1394) and analyzed their efficacy with quantitative PCR (Fig. 2A). All three siRNA fragments reduced hTDE2 mRNA levels in both QGY and SMMC cells with siRNA 895 


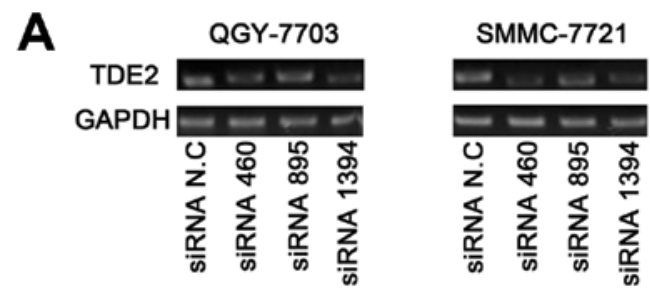

B
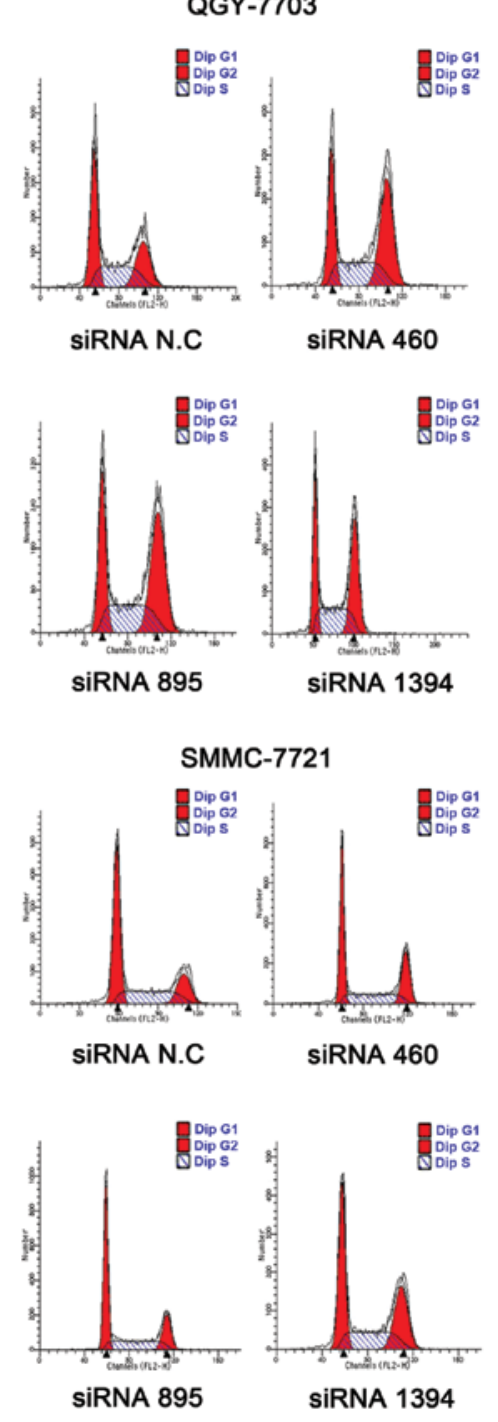
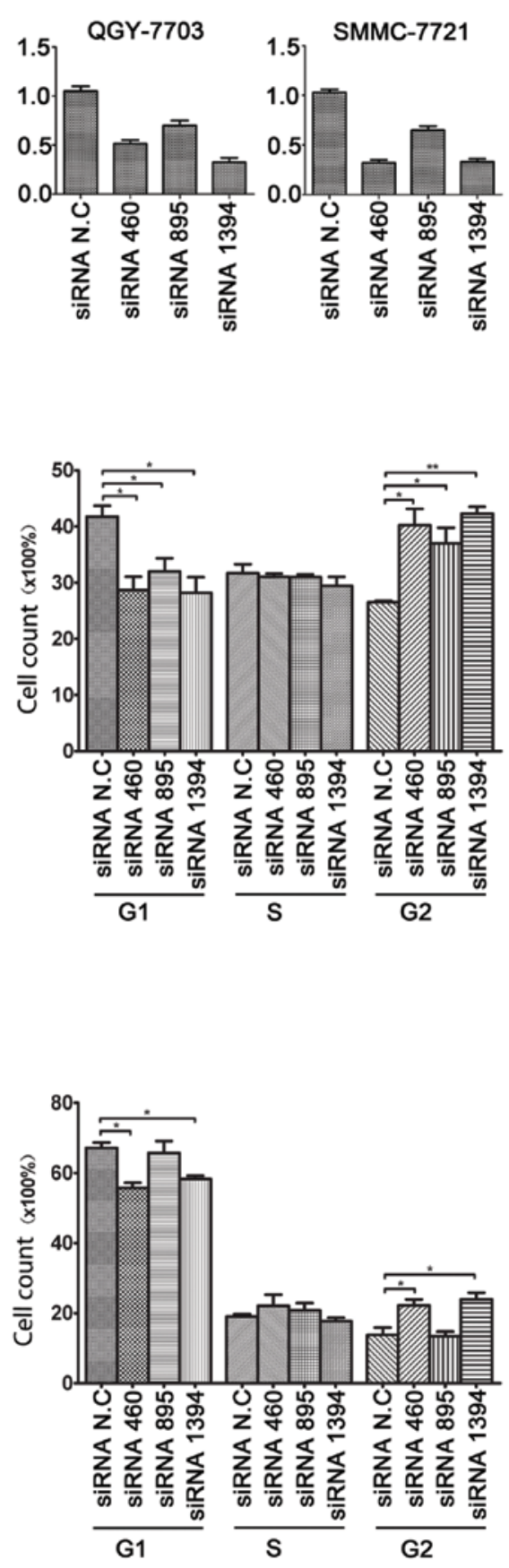

Figure 2. Effect of hTDE2 knockdown on cell cycle arrest. (A) Efficiency of the hTDE2-specific siRNA fragments was assessed by real-time PCR in two HCC cell lines. siRNA NC, non-specific control siRNA fragment. Ratio of $h T D E 2$ mRNA levels is shown on the right, and one tenth of the final PCR products was subjected to agarose electrophoresis (left). (B) Cell cycle analysis of two HCC cell lines by FACS assay. The representative analysis results are shown on the left. Statistical significance is indicated $\left(\mathrm{n}=3,{ }^{*} \mathrm{P}<0.05 ;{ }^{* * *} \mathrm{P}<0.01\right)$.

being relatively less efficient. Then, we utilized FACS assay to detect the cell cycle progression of both HCC cell lines $48 \mathrm{~h}$ after transient transfection of these siRNA fragments. We found that, in both QGY and SMMC, the number of cells in G1 phase decreased and that of G2 cells increased upon hTDE2 knockdown (Fig. 2B). This verified our postulation that reduction of $h T D E 2$ expression may affect $\mathrm{HCC}$ tumor cell proliferation. Furthermore, our cell growth curve by MTT assay also confirmed that downregulation of $h T D E 2$ indeed hindered the growth of tumor cells (Fig. 3A). Three days after siRNA fragment transfection, growth curve of the testing cells started to deviate from that of the controls.

We also performed a colony formation test to further determine the effect of $h T D E 2$ knockdown. With QGY, we found that the colony numbers decreased significantly when the expression of $h T D E 2$ was reduced. This effect was not statistically significant in SMMC cells (Fig. 3B). Our unpublished data further demonstrated that knockdown of $h T D E 2$ 


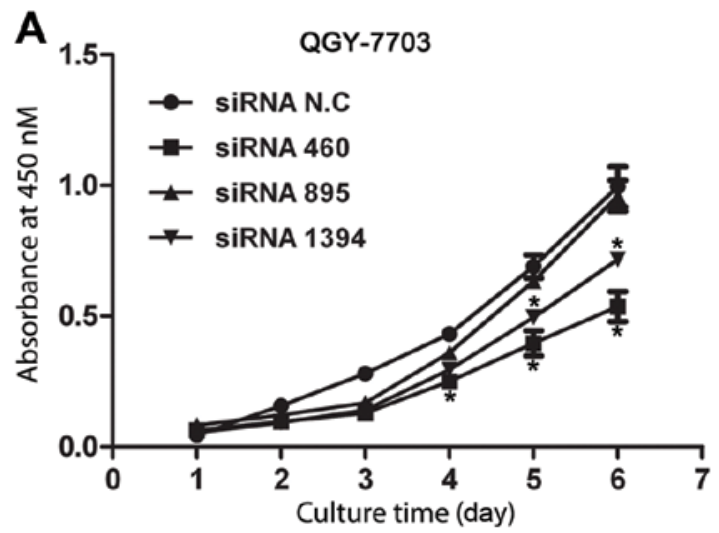

B

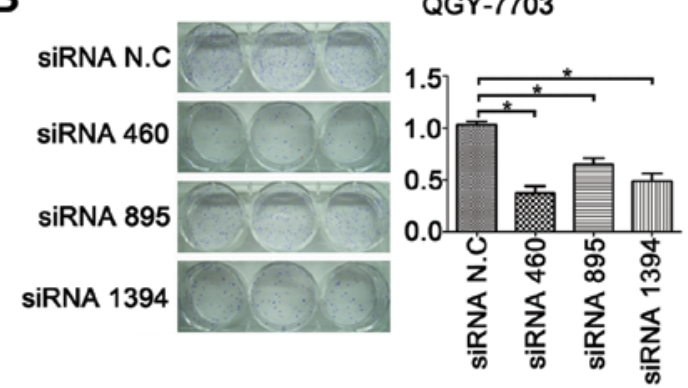

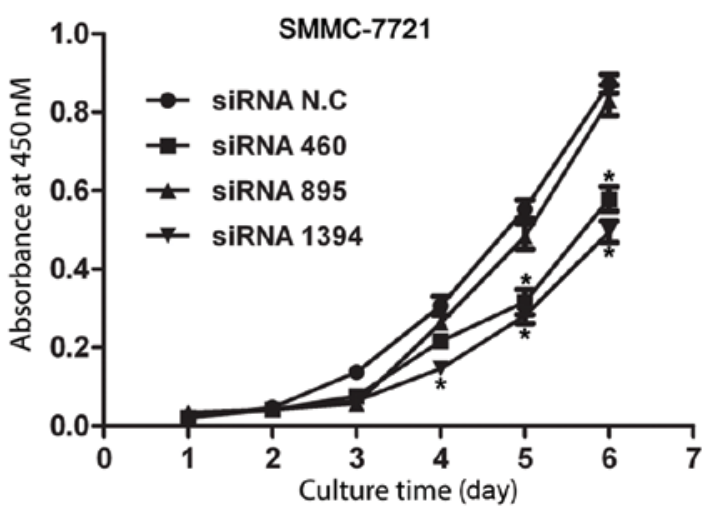

SMMC-7721

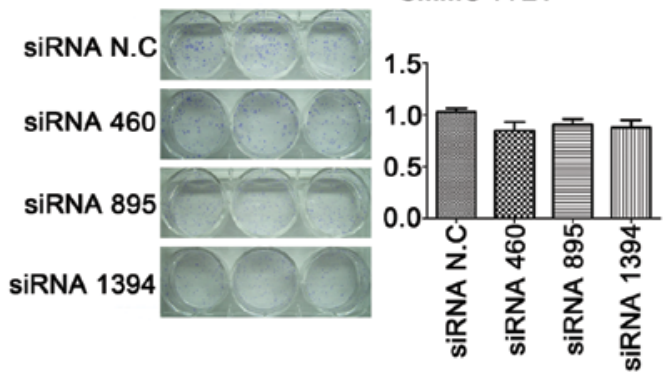

Figure 3. Effect of hTDE2 knockdown on cell growth. (A) Effect of hTDE2 knockdown on cell growth as measured by MTT assay. (B) Effect of hTDE2 knockdown on cell growth as measured by colony formation assay. Statistical significance is indicated $\left(\mathrm{n}=3,{ }^{*} \mathrm{P}<0.05\right)$.

increased apoptosis in QGY, but not in SMMC, which may explain the above inconsistency within the two lines with the colony formation test.

In conclusion, we showed that downregulation of $h T D E 2$ hampered the growth of HCC cells QGY and SMMC, at least partly, via cell cycle retardation.

Knockdown of hTDE2 upregulates p21 and SREBP. We then investigated through which signaling pathway knockdown of TDE2 caused cell cycle arrest. Studies by Inuzuka et al indicated that the transmembrane Serinc/TDE proteins might be involved in the biosynthesis of membrane lipids by facilitating SPT, a rate-limiting enzyme involved in the very early step of multiple membrane lipid biogenesis (7). Therefore, it is reasonable to postulate that reduction of TDE2 expression may affect membrane lipid biogenesis, and this would probably hinder cell cycle progression. p21 functions as a regulator of cell cycle progression at $\mathrm{G} 1$ and $\mathrm{G} 2$, as well as $\mathrm{S}$, and is a transcriptional target of p53. Moreover, p21 transcription is regulated by factors other than $\mathrm{p} 53$. There are multiple cis-acting elements residing in the $5^{\prime}$ UTR of the $p 21$ gene, one of which is the sterol regulatory element (SRE). Combining the biochemical results from Inuzuka et al and our cell cycle results, as well as that SREBPs are pivotal regulators of cellular and membrane lipid homeostasis, we examined whether the expression of SREBPs and p21 could be affected upon TDE2 knockdown.

We first transiently transfected the three $T D E 2$-specific siRNA fragments, as well as the control siRNA, into both QGY and SMMC cell lines. p21 mRNA levels were first analyzed with real-time PCR (Fig. 4A). In both cell lines, p21 was indeed upregulated upon TDE2 knockdown. We also performed western blot analysis to check p21 expression at the protein level, and similar results were reached (Fig. 4B). As mentioned above, we hypothesized the transactivation of $p 21$ here may be through the $S R E$ element. Therefore, expression of the SRE binding factors, SREBPs, was also detected with real-time PCR and western blot analysis. In humans, there are total three SREBP proteins encoded by two genes, SREBPI for SREBP-1a and -1c (resulting from alternative splicing) and $S R E B P 2$ for SREBP2 protein. In our experiments, the expression of SREBP-1c (mRNA and protein) in both QGY and SMMC was very low and undetectable. Therefore, only results for SREBP-1a and SREBP2 are shown. We found that SREBPs (-1a and 2) were upregulated at both the mRNA and the protein level upon TDE2 knockdown, which is consistent with our speculation.

Moreover, the activation of $p 21$ transcription here was p53-independent, with p53 protein level in both QGY and SMMC being unaffected (data not shown). Consistent with this, we also knocked down TDE2 in H2199, a lung cancer cell line with p53 deficiency, and upregulation of $p 21$ was detected as expected (data not shown).

Knockdown of TDE2 transcriptionally activates $p 21$ promoter via SRE element. To further test whether the transcriptional activation of p21 is mediated via the SRE element located in its promoter, we constructed several luciferase reporter plasmids with $p 21$ promoter sequences (full or partial) inserted in front of the luciferase gene (as described in Materials and methods). As shown in Fig. 5A, the promoter sequence of luciferase 
A
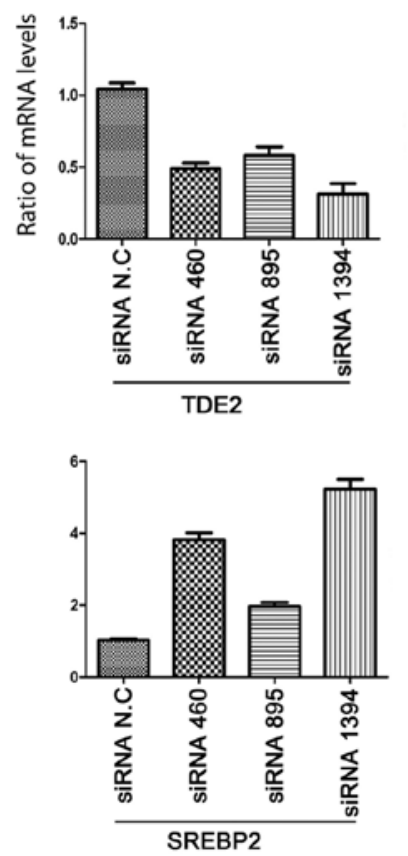

QGY-7703
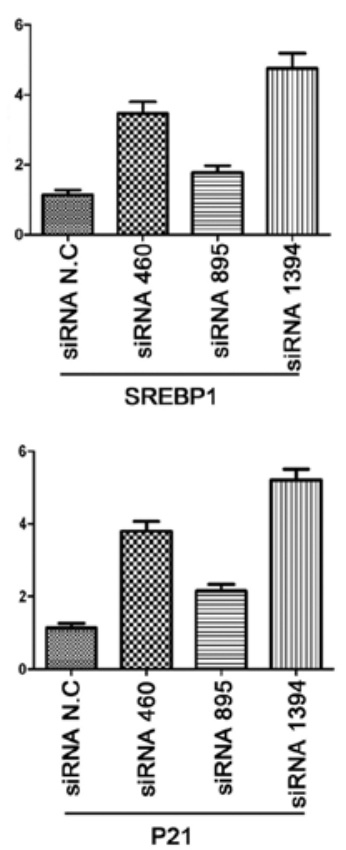

SMMC-7721
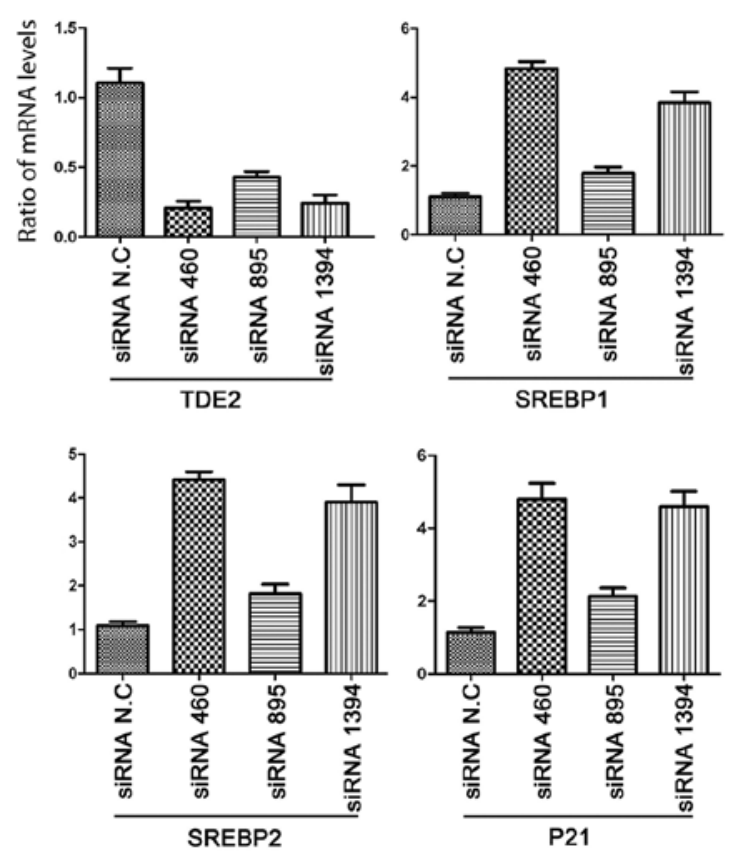

B
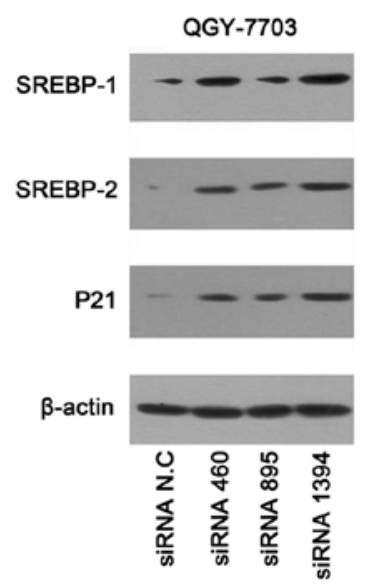

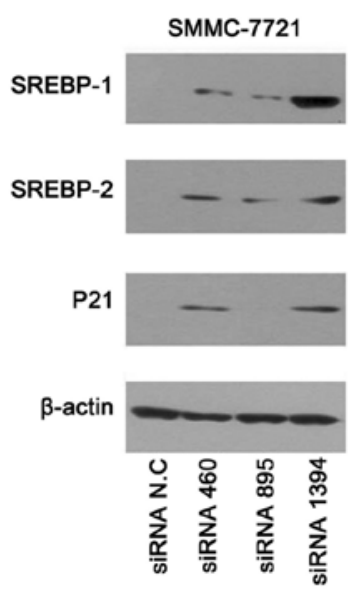

Figure 4. Knockdown of hTDE2 upregulates SREBP1 and p21 expression. (A) mRNA levels of p21 and SREBPs were quantified with real-time PCR in QGY and SMMC. The knockdown effect of the TDE2 siRNA fragments was also assessed. (b) Western blot analysis of SREBP1, SREBP2 and p21 protein levels. $\beta$-actin was used as internal control in western blot analysis while GAPDH was used as control in RT-PCR.

gene in p21-2400-Luc harbors both p53 binding site and SRE element, while that in p21-221-Luc contains only SRE but not the p53-binding site. For both reporter plasmids, we also further mutated its $S R E$ element specifically.

With the dual luciferase report assay, we found that specific knockdown of TDE2 indeed activated p21 promoter. Consistent with previous results, this activation was independent of the p53-binding site. Mutation of SRE sequence completely abolished this transcriptional activation (Fig. 5B and C). We performed the experiments in both QGY and SMMC cells, and obtained similar results.

\section{Discussion}

Serinc/TDE proteins belong to a new transmembrane protein family that is generally tumor differentially expressed. We found that Serinc1/TDE2 was significantly upregulated in hepatocarcinoma tissues and cell lines. However, its precise physiological function remains to be elucidated.

In the present study, we showed that $p 21$ was upregulated upon knockdown of Serincl/TDE2 expression, and this was likely due to SREBP, but not p53. We knocked down Serincl/TDE2 expression in H1299 cells (p53-deficient), and upregulation of $p 21$ was still detected at both the mRNA and protein levels (unpublished data). Our conclusion was further supported by results of the dual luciferase report assay. Moreover, the independence of p53 and SREBP on p21 activation was previously also shown in the p53-deficient Saos-2 cells (20).

In humans, three isoforms of SREBP (-1a, -1c and 2) exist. SREBP2 plays a vital role in the regulation of cholesterol synthesis. While SREBP-1a is involved in the transcription of 
A
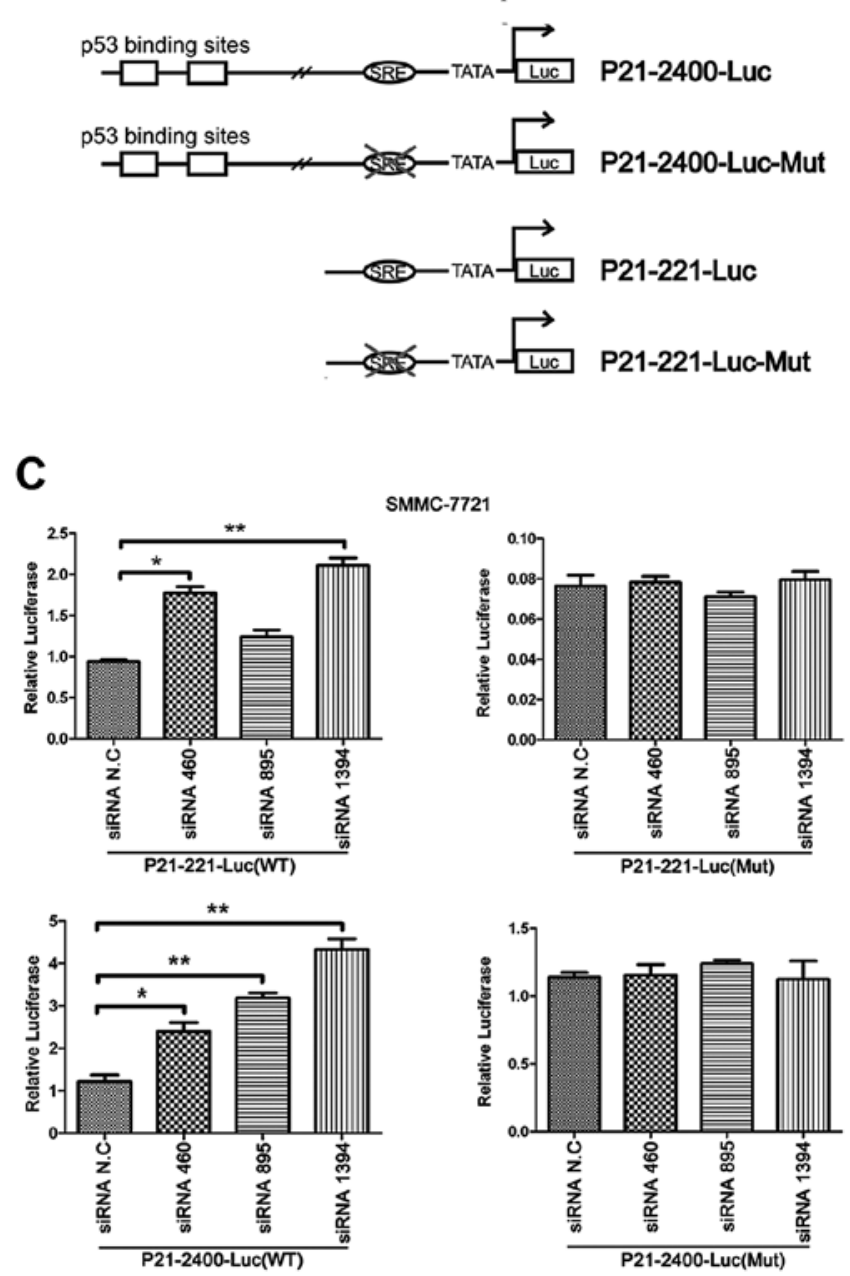

a wide scope of genes involved in cholesterol, fatty acids, and phospholipid synthesis, SREBP-1c has a strong transcriptional activity for enzyme genes involved in fatty acids and triglycerides in lipogenic organs $(17,18)$. Other studies showed that both SREBP-1a and 2 could activate $p 21$ transcription and cause cell growth inhibition. In addition, SREBP-1a could regulate p21 transcription by directly binding to $S R E$ identified in its promoter (20). Although the physiological significance of SREBPs to 21 activation still needs to be clarified, it is postulated that fast growing cells, which require active (membrane) lipid synthesis, may briefly hold cell growth via activating $p 21$ by SREBPs in case of lipid deficiency. Results from the biochemical study of Serinc1/TDE2 (7) and from the present study support the above postulations.

Furthermore, we exogenously expressed hTDE2 in both QGY and SMMC and examined its effect on cell cycle progression. However, compared to the control groups, the experimental groups did not show significant differences in the cell cycle analysis. This may be due to the fact that, under normal conditions, cell cycle progression is limited by factors other than lipid synthesis. Therefore, the overexpression of Serinc1/TDE2 protein will not show considerable effects on cell cycle progression. If we culture the cells in lipid-deficient medium or add drugs to the medium (give cells pressure), the cells may benefit from over-supply of TDE2. Indeed,
B

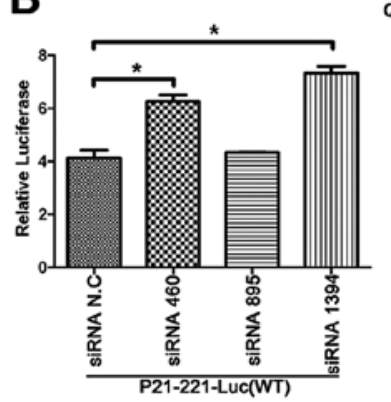

QGY-7703
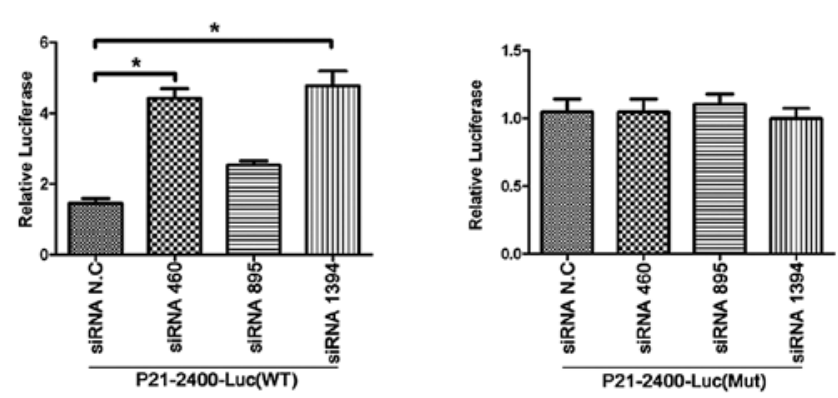

Figure 5. Effect of hTDE2 knockdown on the transcriptional activation activity of p21 promoter. (A) Schematic view of the luciferase reporter constructs containing various lengths of p21 promoter. p21-2400-Luc harbors the promoter site from +73 to -2400 while p21-221-Luc harbors that from +73 to -221 . SRE (oval without $X$ ), wild-type SRE element; SRE (oval with X), SRE element with mutation. (B and C) Upregulation of p21 upon hTDE2 knockdown is $S R E$-dependent. The luciferase reporter assays were performed using both (B) QGY and (C) SMMC cell lines, respectively. Statistical significance is indicated $\left({ }^{*} \mathrm{P}<0.05 ;{ }^{* *} \mathrm{P}<0.01\right)$.

Bossolasco et al (8) showed that cell apoptosis induced by starvation or drug treatment could be partially rescued by TDE1 overexpression.

In conclusion, the present study showed that in both hepatocarcinoma cell lines, downregulation of $h$ Serincl/hTDE2 clearly arrested cell cycle progression. We also observed an increase of both SREBPs and p21 expression upon Serincl/TDE2 knockdown. However, the molecular mechanism underlying this observation still requires detailed investigation. Moreover, although we speculated that the cell cycle arrest observed in the present study might be caused by p21 upregulation via SREBP, we could not exclude other signaling pathways.

\section{Acknowledgements}

The present study was supported by the National Natural Science Foundation of China (30700468).

\section{References}

1. Lebel M and Mes-Masson AM: Establishment and characterization of testicular cell lines from MT-PVLT-10 transgenic mice. Exp Cell Res 213: 12-19, 1994.

2. Liang $P$ and Pardee AB: Differential display of eukaryotic messenger RNA by means of the polymerase chain reaction. Science 257: 967-971, 1992. 
3. Liang P, Averboukh L and Pardee AB: Distribution and cloning of eukaryotic mRNAs by means of differential display: refinements and optimization. Nucleic Acids Res 21: 3269-3275, 1993.

4. Lebel M and Mes-Masson AM: Sequence analysis of a novel cDNA which is overexpressed in testicular tumors from polyomavirus large T-antigen transgenic mice. DNA Seq 5: 31-39, 1994.

5. Grossman TR, Luque JM and Nelson N: Identification of a ubiquitous family of membrane proteins and their expression in mouse brain. J Exp Biol 203: 447-457, 2000.

6. Aoki S, Su O, Li H, Nishikawa K, Ayukawa K, et al: Identification of an axotomy-induced glycosylated protein, AIGP1, possibly involved in cell death triggered by endoplasmic reticulum-golgi stress. J Neurosci 22: 10751-10760, 2002.

7. Inuzuka M, Hayakawa $M$ and Ingi T: Serinc, an activity-regulated protein family, incorporates serine into membrane lipid synthesis. J Biol Chem 280: 35776-35783, 2005.

8. Bossolasco M, Veillette F, Bertrand R and Mes-Masson AM Human TDE1, a TDE1/TMS family member, inhibits apoptosis in vitro and stimulates in vivo tumorigenesis. Oncogene 25: 4549-4558, 2006.

9. Krueger WH, Gonye GE, Madison DL, Murray KE, Kumar M, et al: TPO1, a member of a novel protein family, is developmentally regulated in cultured oligodendrocytes. J Neurochem 69 : 1343-1355, 1997.

10. Bossolasco M, Lebel M, Lemieux N and Mes-Masson AM: The human TDE gene homologue: localization to 20q13.1-13.3 and variable expression in human tumor cell lines and tissue. Mol Carcinog 26: 189-200, 1999.

11. Player A, Gillespie J, Fujii T, Fukuoka J, Dracheva T, et al: Identification of TDE2 gene and its expression in non-small cel lung cancer. Int J Cancer 107: 238-243, 2003.
12. Mizuta K and Warner JR: Continued functioning of the secretory pathway is essential for ribosome synthesis. Mol Cell Biol 14: 2493-2502, 1994

13. Athenstaedt K and Daum G: Tgl4p and Tgl5p, two triacylglycerol lipases of the yeast Saccharomyces cerevisiae are localized to lipid particles. J Biol Chem 280: 37301-37309, 2005.

14. Kurat CF, Natter K, Petschnigg J, Wolinski H, Scheuringer K, et al: Obese yeast: triglyceride lipolysis is functionally conserved from mammals to yeast. J Biol Chem 281: 491-500, 2006.

15. Kurat CF, Wolinski H, Petschnigg J, Kaluarachchi S, Andrews B, et al: $\mathrm{Cdk} 1 / \mathrm{Cdc} 28$-dependent activation of the major triacylglycerol lipase $\mathrm{Tg} 14$ in yeast links lipolysis to cell-cycle progression. Mol Cell 33: 53-63,2009.

16. Futcher B: Tg14 Lipase: a big fat target for cell-cycle entry. Mol Cell 33: 143-144, 2009.

17. Shimano H: Sterol regulatory element-binding proteins (SREBPs): transcriptional regulators of lipid synthetic genes. Prog Lipid Res 40: 439-452, 2001.

18. Horton JD, Goldstein JL and Brown MS: SREBPs: activators of the complete program of cholesterol and fatty acid synthesis in the liver. J Clin Invest 109: 1125-1131, 2002.

19. Nakakuki M, Shimano H, Inoue N, Tamura M, Matsuzaka T, et al: A transcription factor of lipid synthesis, sterol regulatory element-binding protein (SREBP)-1a causes $\mathrm{G}_{1}$ cell-cycle arrest after accumulation of cyclin-dependent kinase (cdk) inhibitors. FEBS J 274: 4440-4452, 2007.

20. Inoue N, Shimano H, Nakakuki M, Matsuzaka T, Nakagawa Y, et al: Lipid synthetic transcription factor SREBP-1a activates p21 $1^{\mathrm{WAF} 1 / C I P 1}$ a universal cyclin-dependent kinase inhibitor. Mol Cell Biol 25: 8938-8947, 2005. 